\title{
HD 114839 - An Am star showing both $\delta$ Scuti and $\gamma$ Dor pulsations discovered through MOST photometry
}

\author{
H. King ${ }^{1}$, J.M. Matthews ${ }^{1}$, J.F. Rowe ${ }^{1}$, \\ C.Cameron ${ }^{1}$, R.Kuschnig ${ }^{1}$, D.B. Guenther ${ }^{2}$, A.F.J. Moffat ${ }^{3}$, S.M. Rucinski ${ }^{4}$, \\ D. Sasselov ${ }^{5}$, G.A.H. Walker ${ }^{1}$, W.W. Weiss ${ }^{6}$ \\ ${ }^{1}$ Department of Physics and Astronomy, University of British Columbia \\ 6224 Agricultural Road, Vancouver, British Columbia, Canada, V6T 1 Z1 \\ 2 Department of Astronomy and Physics, St. Mary's University \\ Halifax, NS B3H 3C3, Canada \\ 3 Département de physique, Université de Montréal \\ C.P. 6128, Succ. Centre-Ville, Montréal, QC H3C 3J7, Canada \\ ${ }^{4}$ David Dunlap Observatory, University of Toronto \\ P.O. Box 360, Richmond Hill, ON L4C 4Y6, Canada \\ ${ }^{5}$ Harvard-Smithsonian Center for Astrophysics \\ 60 Garden Street, Cambridge, MA 02138, USA \\ ${ }^{6}$ Institut für Astronomie, Universität Wien \\ Türkenschanzstrasse 17, A-1180 Wien, Austria
}

\begin{abstract}
Using MOST $^{1}$ (Microvariability and Oscillations of STars) satellite guide star photometry, we have discovered a metallic A star showing hybrid $\mathrm{p}$ - and gmode pulsations. HD 114839 was observed nearly continuously for 10 days in March, 2005. We identify frequencies in three groups: the first centered near 2 cycles/day, in the $\gamma$ Dor pulsation range, and two others near 8 and 20, both in the $\delta$ Scuti range. This is only the fourth known such hybrid pulsator, including another MOST discovery (Rowe et al. 2006).
\end{abstract}

\footnotetext{
${ }^{1}$ MOST is a Canadian Space Agency mission, operated jointly by Dynacon, Inc., and the Universities of Toronto and British Columbia, with assistance from the University of Vienna.
} 


\section{Introduction}

Delta Scuti variables are A-F type stars exhibiting both radial and non-radial p-mode pulsations with periods ranging from about 1 to 5 hours. Gamma Doradus variables are non-radial g-mode pulsators with periods ranging from about 7 hours to 3 days (Kaye et al. 1999), which overlap the red edge of the $\delta$ Scuti instability strip (Handler 1999).

This region of the HR Diagram was searched by Handler \& Shobbrook (2002) for hybrid pulsators exhibiting both p- and g-modes. Their search entailed about $270 \mathrm{hr}$ of photometry of a sample of 26 known and candidate $\gamma$ Dor stars, to look for $\delta$ Scuti-type oscillations. They discovered one hybrid pulsator, HD 209295, a binary system for which the authors argue that excitation of the $\gamma$ Dor g-modes is likely due to tidal interaction. The first known example of a single star showing both $\delta$ Scuti and $\gamma$ Dor pulsations is the metallic A (Am) star HD 8801 (Henry \& Fekel 2005). The authors identified three distinct pairs of frequencies consistent with $\mathrm{g}$ - and $\mathrm{p}$-modes.

It was once thought that all Am stars (which do not have strong global magnetic fields) had binary companions as the measured values of vsini were systematically lower than those for non-peculiar stars in the same part of the HR diagram. The proposed mechanism was tidal breaking in short-period $(P \leq 100$ days) binary systems. Even in the absence of a stabilising magnetic field, the slow rotation might reduce meridional circulation and other atmospheric turbulence enough to allow chemical diffusion to produce the observed abundance anomalies (Abt \& Levy 1985). In Abt \& Levy's study of a sample of $55 \mathrm{Am}$ stars, they found that $75 \%$ showed evidence of spectroscopic binarity. However, the authors estimated that only an additional $8 \%$ of their sample were spectroscopic binaries that were missed due to low orbital inclinations, suggesting that in fact not all Am stars are in binary systems. Another possibility was that slow rotation in single and long-period binary Am stars could be explained by evolutionary effects, but more recent studies (see Henry \& Fekel 2005) suggest that there must be additional factors that have yet to be determined.

Asteroseismology of Am stars could help determine or constrain the physical parameters and evolutionary states of these peculiar stars. Only a small number of pulsation frequencies has been detected in HD 8801 (Henry \& Fekel 2005), limiting its potential for seismic modelling. To explore the possibility of such modelling, other pulsating Am stars with richer eigenspectra must be found.

We report here on just such a discovery, made with the MOST (Microvariability \& Oscillations of STars) space mission (Matthews et al. 2004, Walker et al. 2003). The MOST satellite (launched on 30 June 2003) houses a 15-cm telescope feeding a CCD photometer through a custom broadband optical filter. Its primary mission was to obtain very high-precision photometry of bright 
stars $(V \leq 6)$ to detect pulsations with amplitudes as low as a few $\mu$ mag, with sampling rates of better than one exposure per minute and nearly continuous coverage for weeks at a time. MOST's capabilities were improved and extended after launch to enable astronomical photometry of the fainter $(11 \leq V \leq 7)$ guide stars in the target fields, used to control spacecraft pointing.

During March 2005, HD 114839 was one of the guide stars for observations of the MOST Primary Science target $\beta$ Comae, and low-amplitude oscillations were evident even in the raw photometry. HD 114839 had not attracted much attention prior to these MOST observations. The only accurate stellar parameters for this star available in the literature are from the Hipparcos catalogue: $V=8.46 \pm 0.01, B-V=0.31 \pm 0.01$, spectral type $=$ Am, parallax $\pi=5.04 \pm 1.04$ mas, and $M_{V}=2.06 \pm 0.45$.

\section{Photometry and frequency analysis}

HD 114839 was monitored nearly continuously for about 10 days during 22 31 March 2005, with only one short gap of about 5 hours during the entire run. The exposure time was $25 \mathrm{sec}$, sampled every $30 \mathrm{sec}$. Our final data set contains 27,540 measurements with a duty cycle of $93.5 \%$.

As a guide star, HD 114839 was centred on a $45 \times 45$-pixel subraster of the MOST Startracker CCD. The guide star photometry is almost entirely preprocessed on board the spacecraft (see Walker et al. 2005 for additional details). The mean of the top and bottom rows of the subraster is calculated to provide a threshold value of that mean plus 30 ADU (Analogue-to-Digital Units). The intensities of all pixels in the subraster above that threshold are summed on board, and this value is downloaded to Earth as a flux value for the star, with crude sky subtraction.

The sky background is typically modulated by the 101.4-min orbital period of the MOST satellite, due to stray Earthshine (see Reegen et al. 2005 and Rowe et al. 2006). Since no true sky background measurements are available for MOST guide star photometry, we subtract from the data a running average of the background phased with the MOST orbital period (see Rucinski et al. 2004). This automatically suppresses the MOST orbital frequency and its harmonics in the Fourier spectrum of the data.

The frequency analysis for this star was performed with the program CAPER (Walker et al. 2005) developed by one of the authors (CC). CAPER uses a Discrete Fourier Transform (DFT) to identify frequencies and amplitudes, and then obtains a solution via a simultaneous non-linear least-squares (LevebergMarqardt) fit (Press et al. 1986). We refer the reader to Saio et al. (2006) and Cameron et al. (2006) for more details of CAPER.

The Fourier amplitude spectrum of the HD 114839 photometry is plotted 
in Figure 1, along with the spectral window (which is very clean due to the high duty cycle of the 10 days of MOST data). The initial frequency analysis identified 22 significant frequencies, of which 7 can be attributed to instrumental or orbital artifacts like the stray light modulation mentioned above. Because the photometry is nondifferential, we conservatively reject all power in the DFT below 0.5 cycles/day (c/d). Comparison of the Fourier spectrum of the HD 114839 data to the DFTs of three other guide stars (fainter by about 1 to $1.5 \mathrm{mag}$ ) in the same field shows clearly that the peaks we identify at frequencies above about $1 \mathrm{c} / \mathrm{d}$ are unique and intrinsic to HD 114839.

The frequencies and amplitudes are listed in Table 1.

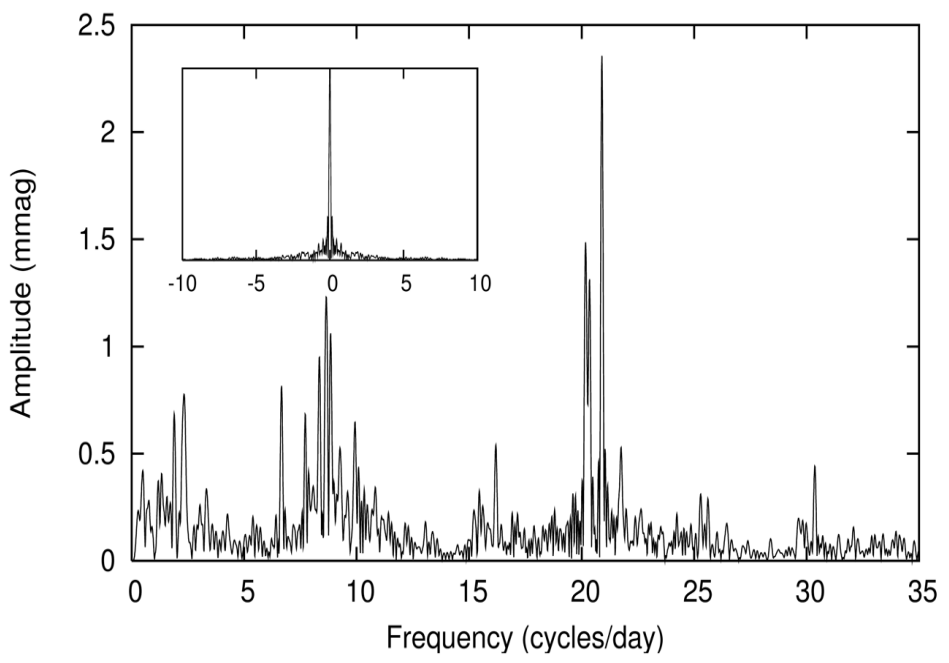

Figure 1: Fourier Spectrum of HD 114839, after removal of known instrumental artifacts. Inset is the window function for the highest peak in the spectrum.

\section{A new hybrid pulsator}

We identify 15 frequencies in HD 114839 with a signal-to-noise $(S / N)$ level in amplitude above 3.8. Four of these frequencies are between 1 and $2.5 \mathrm{c} / \mathrm{d}$, consistent with $\gamma$ Dor g-mode pulsations, while the remaining frequencies are $\delta$ Scuti-type p-modes between about 6.5 and $22 \mathrm{c} / \mathrm{d}$. The p-mode frequencies are grouped into two ranges, 7 frequencies around $8 \mathrm{c} / \mathrm{d}$ and 4 around 21 c/d. We note that the frequencies found by Henry \& Fekel (2005) in HD 8801 
Table 1: Identified frequencies for the star HD 114839

\begin{tabular}{|c|c|c|}
\hline${\text { Frequency }\left(\text { day }^{-1}\right)}^{-}$ & Amplitude(mmag) & $\mathrm{S} / \mathrm{N}$ \\
\hline 1.3412 & $0.473 \pm 0.004$ & 3.87 \\
1.8905 & $0.637 \pm 0.004$ & 4.25 \\
2.2609 & $0.609 \pm 0.004$ & 3.95 \\
2.3376 & $0.771 \pm 0.004$ & 4.75 \\
6.6678 & $0.794 \pm 0.004$ & 4.87 \\
7.7152 & $0.624 \pm 0.004$ & 5.22 \\
8.3411 & $0.910 \pm 0.004$ & 6.94 \\
8.6477 & $1.210 \pm 0.004$ & 8.84 \\
8.8649 & $1.000 \pm 0.004$ & 8.00 \\
9.2864 & $0.528 \pm 0.004$ & 4.32 \\
9.9378 & $0.593 \pm 0.004$ & 4.97 \\
20.1822 & $1.520 \pm 0.004$ & 9.58 \\
20.3483 & $1.450 \pm 0.004$ & 10.02 \\
20.8976 & $2.260 \pm 0.004$ & 15.23 \\
21.7534 & $0.461 \pm 0.004$ & 4.72 \\
\hline
\end{tabular}

- another Am star - were clustered in a similar fashion, near 3, 8 and 20 c/d. We cannot establish without doubt that HD 114839 is a single star, lacking spectroscopic confirmation, but the possible parallels with HD 8801 are intriguing. In any event, this discovery and that by Rowe et al. (2006) reported in this issue together double the number of known hybrid pulsators, and possibly triple the number of single stars which exhibit both $\delta$ Scuti and $\gamma$ Doradus modes simultaneously.

Acknowledgments. JMM, DBG, AFJM, SR, and GAHW are supported by funding from the Natural Sciences and Engineering Research Council (NSERC) Canada. RK is funded by the Canadian Space Agency. WWW is supported by the Austrian Science Promotion Agency (FFG - MOST) and the Austrian Science Funds (FWF - P17580).

\section{References}

Abt, H.A, \& Levy, S.G. 1985, ApJS, 59, 229

Cameron, C., Matthews, J.M., Rowe, J.F., et al. 2006, CoAst, 148, 58

ESA 1997, The HIPPARCOS and TYCHO catalogues, Astrometric and photometric star catalogues derived from the ESA HIPPARCOS Space Astrometry Mission (ESA Publikations Division, Noordwijk, Netherlands), ESA SP Ser., 1200 
Handler, G. 1999, MNRAS, 309, L19

Handler, G., \& Shobbrook, R.R. 2002, MNRAS, 333, 251

Henry, G. \& W. Fekel, F. C. 2005, AJ, 129, 2026

Kaye, A.B., et al. 1999, PASP, 116, 558

Matthews, J.M., et al. 2004, Nature, 430, 51-53

Press, W.H., et al. 1986, Numerical Recipes in Fortran 77 (Cambridge University

Press), 678

Reegen, P., et al. 2005, MNRAS, 367, 1417

Rowe, J.F., Matthews, J.M., Cameron, C., et al. 2006, CoAST, CoAst, 148, 34

Rucinski, S.M., et al. 2004, PASP, 116, 1093

Saio, H., et al. 2006, astro-ph/0606712

Walker, G. A., et al. 2003, PASP, 115, 1023

Walker, G. A., et al. 2005, ApJ, 635, L77 